\title{
THE BEST CONSTANT IN A FRACTIONAL HARDY INEQUALITY
}

\author{
KRZYSZTOF BOGDAN AND BARTŁOMIEJ DYDA
}

\begin{abstract}
We prove an optimal Hardy inequality for the fractional Laplacian on the half-space.
\end{abstract}

\section{Main Result And Discussion}

Let $0<\alpha<2$ and $d=1,2, \ldots$. The purpose of this note is to prove the following Hardy-type inequality in the half-space $D=\left\{x=\left(x_{1}, \ldots, x_{d}\right) \in \mathbb{R}^{d}: x_{d}>0\right\}$.

Theorem 1. For every $u \in C_{c}(D)$,

$$
\frac{1}{2} \int_{D} \int_{D} \frac{(u(x)-u(y))^{2}}{|x-y|^{d+\alpha}} d x d y \geq \kappa_{d, \alpha} \int_{D} u^{2}(x) x_{d}^{-\alpha} d x
$$

where

$$
\kappa_{d, \alpha}=\frac{\pi^{\frac{d-1}{2}} \Gamma\left(\frac{1+\alpha}{2}\right)}{\Gamma\left(\frac{\alpha+d}{2}\right)} \frac{B\left(\frac{1+\alpha}{2}, \frac{2-\alpha}{2}\right)-2^{\alpha}}{\alpha 2^{\alpha}},
$$

and (11) fails to hold for some $u \in C_{c}(D)$ if $\kappa_{d, \alpha}$ is replaced by a bigger constant.

Here $B$ is the Euler beta function, and $C_{c}(D)$ denotes the class of all the continuous functions $u: \mathbb{R}^{d} \rightarrow \mathbb{R}$ with compact support in $D$. On the right-hand side of (1) we note the infinite measure $x_{d}^{-\alpha} d x$, where $x_{d}$ equals the distance of $x=\left(x_{1}, \ldots, x_{d}\right) \in D$ to the complement of $D$. Analogous Hardy inequalities, involving the distance to the complement of rather general domains, and arbitrary positive exponents of integrability of functions $u$, were proved with rough constants in [16] (see also [33, 14, 17]). Thus the focus in Theorem[1] is on optimality of $\kappa_{d, \alpha}$. We note that $\kappa_{d, 1}=0$ and $\kappa_{d, \alpha}>0$ if $\alpha \neq 1$ (see the proof of Lemma 2).

Theorem 1 may be viewed as an application of ideas of Ancona 11 and Fitzsimmons [19. Indeed, consider the Dirichlet form $\mathcal{E}$, with domain $\operatorname{Dom}(\mathcal{E})$, and the generator $\mathcal{L}$, with domain $\operatorname{Dom}(\mathcal{L})$, of a symmetric Markov process (22], 35], 29]), and a function $w>0$, and a measure $\nu \geq 0$ on the state space. The following result was proved by Fitzsimmons in [19.

$$
\text { If } \mathcal{L} w \leq-w \nu \text { then } \mathcal{E}(u, u) \geq \int u^{2} d \nu, \quad u \in \operatorname{Dom}(\mathcal{E}) .
$$

Thus, every superharmonic function $w$ (i.e. $w \geq 0$ such that $\mathcal{L} w \leq 0$ ) yields a Hardy-type inequality with integral weight $\nu=-\mathcal{L} w / w$. For instance, in the proof

2000 Mathematics Subject Classification: Primary 26D10, secondary 31C25, 31B25, 46E35.

Key words and phrases: fractional Hardy inequality, best constant, half-space, fractional Laplacian, censored stable process.

Research partially supported by KBN 1 P03A 02629

Date: May 28, 2018. 
of Theorem 1 we will use $w(x)=x_{d}^{(\alpha-1) / 2}$. Full details of (3), and a converse result are given in [19, Theorem 1.9]. Recall that

$$
\mathcal{E}(u, v)=-(L u, v), \quad \text { if } u \in \operatorname{Dom}(\mathcal{L}), v \in \operatorname{Dom}(\mathcal{E}),
$$

(22], 35]). Therefore, equality holds in (3) if $u=w \in \operatorname{Dom}(\mathcal{L})$, see [19, (1.13.c)], (91). If $w \notin \operatorname{Dom}(\mathcal{L})$, or $\mathcal{L} w$ does not belong to the underlying $L^{2}$ space, then, as we shall see, the optimality of $\nu=-\mathcal{L} w / w$ critically depends on the choice of $w$.

According to [7, the Dirichlet form of the censored $\alpha$-stable process in $D$ is

$$
\mathcal{C}(u, v)=\frac{1}{2} \mathcal{A}_{d,-\alpha} \int_{D} \int_{D} \frac{(u(x)-u(y))(v(x)-v(y))}{|x-y|^{d+\alpha}} d x d y,
$$

with core $C_{c}^{\infty}(D)$ (smooth functions in $C_{D}(D)$ ), the Lebesgue measure as the reference measure, and the following regional fractional Laplacian on $D$ as the generator ([7, (3.12)], [25, 26]):

$$
\Delta_{D}^{\alpha / 2} u(x)=\mathcal{A}_{d,-\alpha} \lim _{\varepsilon \rightarrow 0^{+}} \int_{D \cap\{|y-x|>\varepsilon\}} \frac{u(y)-u(x)}{|x-y|^{d+\alpha}} d y .
$$

Here $\mathcal{A}_{d,-\alpha}=\Gamma((d+\alpha) / 2) /\left(2^{-\alpha} \pi^{d / 2}|\Gamma(-\alpha / 2)|\right)$, Clearly, (11) is equivalent to

$$
\mathcal{C}(u, u) \geq \mathcal{A}_{d,-\alpha} \kappa_{d, \alpha} \int_{D} u^{2}(x) x_{d}^{-\alpha} d x .
$$

Recall that the Dirichlet form of the stable process killed when leaving $D$ is

$$
\mathcal{K}(u, v)=\frac{1}{2} \mathcal{A}_{d,-\alpha} \int_{\mathbb{R}^{d}} \int_{\mathbb{R}^{d}} \frac{(u(x)-u(y))(v(x)-v(y))}{|x-y|^{d+\alpha}} d x d y,
$$

with core $C_{c}^{\infty}(D)$, the Lebesgue measure as the reference measure, and the fractional Laplacian (on $\mathbb{R}^{d}$ ) as the generator,

$$
\Delta^{\alpha / 2} u(x)=\mathcal{A}_{d,-\alpha} \lim _{\varepsilon \rightarrow 0^{+}} \int_{\mathbb{R}^{d} \cap\{|y-x|>\varepsilon\}} \frac{u(y)-u(x)}{|x-y|^{d+\alpha}} d y
$$

(see, e.g., [7]). Decomposing $\mathbb{R}^{d}=D \cup D^{c}$, one obtains

$$
\mathcal{K}(u, u)=\mathcal{C}(u, u)+\int_{D} u^{2}(x) \kappa_{D}(x) d x, \quad u \in C_{c}^{\infty}(D),
$$

where (the density of the killing measure for $D$ is)

$$
\kappa_{D}(x)=\int_{D^{c}} \mathcal{A}_{d,-\alpha}|x-y|^{-d-\alpha} d y=\frac{1}{\alpha} \mathcal{A}_{d,-\alpha} \frac{\pi^{\frac{d-1}{2}} \Gamma\left(\frac{1+\alpha}{2}\right)}{\Gamma\left(\frac{\alpha+d}{2}\right)} x_{d}^{-\alpha},
$$

see [7. (2.3), (5.4)-(5.6)]. It follows from (44) and Theorem [1 that

$$
\begin{aligned}
\mathcal{C}(u, u) & \geq \mathcal{A}_{d,-\alpha}\left(\kappa_{d, \alpha}+\frac{1}{\alpha} \frac{\pi^{\frac{d-1}{2}} \Gamma\left(\frac{1+\alpha}{2}\right)}{\Gamma\left(\frac{\alpha+d}{2}\right)}\right) \int_{D} u^{2}(x) x_{d}^{-\alpha} d x \\
& =\frac{\Gamma^{2}\left(\frac{1+\alpha}{2}\right)}{\pi} \int_{D} u^{2}(x) x_{d}^{-\alpha} d x,
\end{aligned}
$$

for all $u \in C_{c}^{\infty}(D)$, and the constant $\Gamma^{2}\left(\frac{1+\alpha}{2}\right) / \pi$ is the best possible.

We like to note that in some respects, the censored stable process is a better analogue of the killed Brownian motion than the killed stable process is (see [14, 7, 32, and [37, 31]). We suggest the former as a possible setup for studying Dirichlet boundary value problems for non-local integro-differential operators and the corresponding stochastic processes $\left(\left[\underline{30},[38]\right.\right.$ ) on subdomains of $\mathbb{R}^{d}([3])$, beyond the 
"convolutional" case of the whole of $\mathbb{R}^{d}([21,4])$. In this connection, we refer to 25, 26, 24, for Green-type formulas for the censored process.

The reader interested in fractional Hardy inequalities may consult [34, 27, 33, 14, 16, 17. In particular, (11) improves a part of the (one-dimensional) result given in [33, Theorem 2]. The fractional Hardy inequality on the whole of $\mathbb{R}^{d}$ is known as Hardy-Rellich inequality, and the best constant in this inequality was calculated in 28, 39 (see also [4 for Pitt's inequality). As seen in [16, the asymptotics of the measure dist $\left(x, D^{c}\right)^{-\alpha} d x$ agrees well with the homogeneity of the kernel $|y-x|^{-d-\alpha}$ in (11). Noteworthy, if $\alpha \leq 1$ and $D$ is a bounded Lipschitz domain, then the best constant in (11) is zero ([16]).

We like to make a few further remarks. Theorem 1 and the results obtained to date for Laplacian and fractional Laplacian suggest possible strengthenings to weights with additional terms of lower-order boundary asymptotics ([11, 23, 4]), and extensions to other specific or more general domains ([36, 4]). To discuss the latter problem, we consider open $\Omega \subset D$, and its Hardy constant, $\kappa(\Omega)$, defined as the largest number such that

$$
\frac{1}{2} \int_{\Omega} \int_{\Omega} \frac{(u(x)-u(y))^{2}}{|x-y|^{d+\alpha}} d x d y \geq \kappa(\Omega) \int_{\Omega} \frac{u^{2}(x)}{\operatorname{dist}\left(x, \Omega^{c}\right)^{\alpha}} d x, \quad u \in C_{c}(\Omega) .
$$

Note that $\kappa(\Omega)>0$ if $\Omega$ is a bounded Lipschitz domain and $\alpha>1$ ([16]). Let $u \in C_{c}(\Omega) \subset C_{c}(D)$. We have

$$
\frac{1}{2} \int_{\Omega} \int_{\Omega} \frac{(u(x)-u(y))^{2}}{|x-y|^{d+\alpha}} d x d y \leq \frac{1}{2} \int_{D} \int_{D} \frac{(u(x)-u(y))^{2}}{|x-y|^{d+\alpha}} d x d y,
$$

and

$$
\int_{\Omega} \frac{u^{2}(x)}{\operatorname{dist}\left(x, \Omega^{c}\right)^{\alpha}} d x \geq \int_{D} \frac{u^{2}(x)}{x_{d}^{\alpha}} d x
$$

thus $\kappa(\Omega) \leq \kappa_{d, \alpha}$. We conjecture that $\kappa(\Omega)=\kappa_{d, \alpha}$ for $\alpha \in(1,2)$ and convex $\Omega$, see 36. Theorem 11] for case of the Dirichlet of Laplacian.

Examining (2) we see that $\kappa_{d, \alpha} \rightarrow \infty$ if $\alpha \rightarrow 2$. This corresponds to the fact that the only function $u \in C_{c}(\Omega)$ for which the left hand side of (1) is finite for $\alpha=2$ is the zero function, see [12, 16]. However, $\mathcal{A}_{d,-\alpha} \kappa_{d, \alpha} \rightarrow 1 / 4$ and $\Gamma^{2}\left(\frac{1+\alpha}{2}\right) / \pi \rightarrow 1 / 4$ as $\alpha \rightarrow 2$, an agreement with the classical Hardy inequality for Laplacian (11]) related to the fact that for $u \in C_{c}^{\infty}(D), \Delta_{D}^{\alpha / 2} u \rightarrow \Delta u$ and $\mathcal{C}(u, u) \rightarrow-\int \Delta u(x) u(x) d x=$ $\int|\nabla u(x)|^{2} d x$ as $\alpha \rightarrow 2$ (the latter holds by Taylor's expansion of order 2 , and a similar result is valid for $\mathcal{K})$. For the vast literature concerning optimal weights and constants in the classical Hardy inequalities, and their applications we refer to [5, 15, 11, 20, 23, 18, 2.

Our primary motivation to study Hardy inequalities for non-local Dirichlet forms stems from the fact that the converse of (3) stated in [19, Theorem 1.9] allows for a construction of superharmonic functions, or barriers ([1]), when a Hardy inequality is given. These functions may then be used to investigate transience and boundary behavior of the underlying Markov processes (11, 7], 17, [14]). In particular, we expect that the results of [16, 17. may be used to obtain, for the anisotropic stable ([10, 9]) censored processes, the ruin probabilities generalizing [7, Theorem 5.10], and to develop the boundary potential theory on Lipschitz domains ( $7,26,24)$ in analogy with those of the killed stable processes $([8,13,6])$. We also like to mention the connection of optimal Hardy inequalities with critical Schrödinger perturbations and the so-called ground state representation [21]. 
Despite the general context mentioned above, the paper is essentially selfcontained and purely analytic. In particular we directly derive Fitzsimmons' ratio measure by a simple manipulation with quadratic expressions, (9), not unrelated to the ground state representation of [21, (4.2)]. Theorem 1 is proved below in this section. In Section 2 we calculate auxiliary integrals.

In what follows, $|x|=\left(x_{1}^{2}+\cdots+x_{d}^{2}\right)^{1 / 2}$ denotes the Euclidean norm of $x=$ $\left(x_{1}, \ldots, x_{d}\right) \in \mathbb{R}^{d}$, and $B(x, r)$ denotes the Euclidean ball of radius $r>0$ centered at $x$. For $d \geq 2$ we occasionally write $x=\left(x^{\prime}, x_{d}\right)$, where $x^{\prime}=\left(x_{1}, \ldots, x_{d-1}\right)$, and we let $\left\|x^{\prime}\right\|=\max _{k=1, \ldots, d-1}\left|x_{k}\right|$, the supremum norm on $\mathbb{R}^{d-1}$.

Proof of Theorem 1, For $u, v \in C_{c}^{\infty}(D)$ we define (Dirichlet form)

$$
\mathcal{E}(u, v)=\frac{1}{2} \int_{D} \int_{D} \frac{(u(x)-u(y))(v(x)-v(y))}{|x-y|^{d+\alpha}} d x d y,
$$

and (its generator)

$$
\mathcal{L} u(x)=\lim _{\varepsilon \rightarrow 0^{+}} \int_{D \cap\{|y-x|>\varepsilon\}} \frac{u(y)-u(x)}{|x-y|^{d+\alpha}} d y,
$$

so that $\mathcal{A}_{d,-\alpha} \mathcal{E}=\mathcal{C}, \mathcal{A}_{d,-\alpha} \mathcal{L}=\Delta_{D}^{\alpha / 2}$, and $\mathcal{E}(u, u)$ equals the left-hand side of (11).

Let $p \in(-1, \alpha), x=\left(x_{1}, \ldots, x_{d}\right) \in D$,

$$
w_{p}(x)=x_{d}^{p} .
$$

By [7, (5.4) and (5.5)],

$$
\mathcal{L} w_{p}(x)=\gamma(\alpha, p) \frac{\pi^{\frac{d-1}{2}} \Gamma\left(\frac{1+\alpha}{2}\right)}{\Gamma\left(\frac{\alpha+d}{2}\right)} x_{d}^{-\alpha} w_{p}(x),
$$

where the (absolutely convergent) integral

$$
\gamma(\alpha, p)=\int_{0}^{1} \frac{\left(t^{p}-1\right)\left(1-t^{\alpha-p-1}\right)}{(1-t)^{1+\alpha}} d t
$$

is negative if $p(\alpha-p-1)>0$. Guided by the discussion in Section 1 we let

$$
\nu(x)=\frac{-\mathcal{L} w_{p}(x)}{w_{p}(x)}=-\gamma(\alpha, p) \frac{\pi^{\frac{d-1}{2}} \Gamma\left(\frac{1+\alpha}{2}\right)}{\Gamma\left(\frac{\alpha+d}{2}\right)} x_{d}^{-\alpha} .
$$

Since, for each $t \in(0,1)$, the function

$$
p \mapsto \frac{\left(t^{p}-1\right)\left(1-t^{\alpha-p-1}\right)}{(1-t)^{1+\alpha}}
$$

is convex and symmetric with respect to $(\alpha-1) / 2$, therefore $p \mapsto \gamma(\alpha, p)$ has a non-positive minimum at $p=(\alpha-1) / 2$. By Lemma 2 below, (8), and (3), we obtain (11) for $u \in C_{c}^{\infty}(D) \subset \operatorname{Dom}(\mathcal{C})$, with $\kappa_{d, \alpha}$ given by (2). The case of general $u \in C_{c}(D)$ is obtained by an approximation.

Since the setups of [19] and [7] are rather complex, we like to give the following elementary proof of (1). Let $w=w_{(\alpha-1) / 2}, u \in C_{c}(D), x, y \in D$. We have

$$
\begin{aligned}
(u(x)-u(y))^{2} & +u^{2}(x) \frac{w(y)-w(x)}{w(x)}+u^{2}(y) \frac{w(x)-w(y)}{w(y)} \\
& =w(x) w(y)[u(x) / w(x)-u(y) / w(y)]^{2} \geq 0 .
\end{aligned}
$$


We integrate (91) against the symmetric measure $1_{|y-x|>\varepsilon}|x-y|^{-d-\alpha} d x d y$, and we let $\varepsilon \rightarrow 0^{+}$. According to the calculations above,

$$
\begin{aligned}
\frac{1}{2} \int_{D} \int_{D} \frac{(u(x)-u(y))^{2}}{|x-y|^{d+\alpha}} d x d y & \geq \int_{D} u^{2}(x) \lim _{\substack{\varepsilon \rightarrow 0^{+} \\
\{y \in D:|y-x|>\varepsilon\}}} \frac{w(x)-w(y)}{|y-x|^{d+\alpha}} d y \frac{d x}{w(x)} \\
& =\kappa_{d, \alpha} \int_{D} u^{2}(x) x_{d}^{-\alpha} d x .
\end{aligned}
$$

To complete the proof we will verify the optimality of $\kappa_{d, \alpha}$. In what follows we denote $\mathbf{p}=\frac{\alpha-1}{2}$. If $\alpha \geq 1$ then we consider functions $v_{n}$ such that

(i) $v_{n}=1$ on $\left[-n^{2}, n^{2}\right]^{d-1} \times\left[\frac{1}{n}, 1\right]$,

(ii) $\operatorname{supp} v_{n} \subset\left[-n^{2}-1, n^{2}+1\right]^{d-1} \times\left[\frac{1}{2 n}, 2\right]$,

(iii) $0 \leq v_{n} \leq 1,\left|\nabla v_{n}(x)\right| \leq c x_{d}^{-1}$ and $\left|\nabla^{2} v_{n}(x)\right| \leq c x_{d}^{-2}$ for $x \in D$.

If $\alpha<1$ then we stipulate

(i') $v_{n}=1$ on $\left[-n^{2}, n^{2}\right]^{d-1} \times[1, n]$,

(ii') $\operatorname{supp} v_{n} \subset\left[-n^{2}-n, n^{2}+n\right]^{d-1} \times\left[\frac{1}{2}, 2 n\right]$,

(iii) $0 \leq v_{n} \leq 1,\left|\nabla v_{n}(x)\right| \leq c x_{d}^{-1}$ and $\left|\nabla^{2} v_{n}(x)\right| \leq c x_{d}^{-2}$ for $x \in D$.

We define (for any $\alpha \in(0,2)$ ),

$$
u_{n}(x)=v_{n}(x) x_{d}^{\mathbf{p}}
$$

We have

$$
\int_{D} \frac{u_{n}(x)^{2}}{x_{d}^{\alpha}} d x \geq \int_{\left\{x:\left\|x^{\prime}\right\| \leq n^{2}, \frac{1}{n}<x_{d}<1\right\}} \frac{x_{d}^{2 \mathbf{p}}}{x_{d}^{\alpha}} d x=\left(2 n^{2}\right)^{d-1} \log n .
$$

Thus, by Lemma 4 below, $\kappa_{d, \alpha}$ may not be replaced in (1) by a bigger constant.

\section{APPENDIX}

Lemma 2. For $0<\alpha<2$,

$$
\gamma\left(\alpha, \frac{\alpha-1}{2}\right)=-\frac{1}{\alpha}\left[B\left(\frac{1+\alpha}{2}, \frac{2-\alpha}{2}\right) 2^{-\alpha}-1\right] .
$$

Proof. Since

$$
\gamma(\alpha, p)=\int_{0}^{1} \frac{t^{p}-t^{\alpha-1}-1+t^{\alpha-p-1}}{(1-t)^{1+\alpha}} d t,
$$

we are led to considering

$$
B_{\kappa}(a, b)=\int_{0}^{\kappa} t^{a-1}(1-t)^{b-1} d t .
$$

Here and below $a>0, b>-2$, and $0 \leq \kappa<1$. We will also assume that $b \neq 0,1$.

Using $t^{a-1}=t^{a-1}(1-t)+t^{a}$, and integration by parts, we get

$$
B_{\kappa}(a, b)=\frac{a+b}{b} B_{\kappa}(a, b+1)-\frac{1}{b} \kappa^{a}(1-\kappa)^{b},
$$

hence

$$
B_{\kappa}(a, b)=\frac{a+b}{b}\left(\frac{a+b+1}{b+1} B_{\kappa}(a, b+2)-\frac{1}{b+1} \kappa^{a}(1-\kappa)^{b+1}\right)-\frac{1}{b} \kappa^{a}(1-\kappa)^{b} .
$$


Clearly, $\gamma(\alpha, p)=\lim _{\kappa \rightarrow 1^{-}}\left[B_{\kappa}(p+1,-\alpha)-B_{\kappa}(\alpha,-\alpha)-B_{\kappa}(1,-\alpha)+B_{\kappa}(\alpha-p,-\alpha)\right]$. For $\alpha \neq 1$ we have,

$$
\begin{aligned}
& B_{\kappa}(p+1,-\alpha)-B_{\kappa}(\alpha,-\alpha)-B_{\kappa}(1,-\alpha)+B_{\kappa}(\alpha-p,-\alpha)=\frac{1}{\alpha(\alpha-1)} \times \\
& \left\{(p+1-\alpha)(p+1-\alpha+1) B_{\kappa}(p+1,2-\alpha)-(\alpha-\alpha)(\alpha-\alpha+1) B_{\kappa}(\alpha, 2-\alpha)\right. \\
& \left.-(1-\alpha)(1-\alpha+1) B_{\kappa}(1,2-\alpha)+(\alpha-p-\alpha)(\alpha-p-\alpha+1) B_{\kappa}(\alpha-p, 2-\alpha)\right\} \\
& +\frac{(1-\kappa)^{1-\alpha}}{\alpha(\alpha-1)}\left[-(p+1-\alpha) \kappa^{p+1}+(\alpha-\alpha) \kappa^{\alpha}+(1-\alpha) \kappa^{1}-(\alpha-p-\alpha) \kappa^{\alpha-p}\right] \\
& +\frac{(1-\kappa)^{-\alpha}}{-\alpha}\left[-\kappa^{p+1}+\kappa^{\alpha}+\kappa^{1}-\kappa^{\alpha-p}\right] .
\end{aligned}
$$

All expressions in the square brackets, and their derivative, vanish at $\kappa=1$. Thus, they do not contribute to the limit as $\kappa \rightarrow 1$. For $\alpha \neq 1$ we get

$$
\begin{aligned}
\gamma(\alpha, p)= & \frac{1}{\alpha(\alpha-1)}\{(p+1-\alpha)(p+2-\alpha) B(p+1,2-\alpha) \\
& -(1-\alpha)(2-\alpha) B(1,2-\alpha)+p(p-1) B(\alpha-p, 2-\alpha)\} .
\end{aligned}
$$

By the duplication formula $\Gamma(2 z)=(2 \pi)^{-1 / 2} 2^{2 z-1 / 2} \Gamma(z) \Gamma(z+1 / 2)$ with $2 z=2-\alpha$, for $p=(\alpha-1) / 2$, this equals

$$
\begin{aligned}
& \frac{1}{\alpha}\left[-\frac{3-\alpha}{2} B\left(\frac{\alpha+1}{2}, 2-\alpha\right)+1\right]=\frac{1}{\alpha}\left[-\Gamma\left(\frac{\alpha+1}{2}\right) \Gamma(2-\alpha) / \Gamma\left(\frac{3-\alpha}{2}\right)+1\right] \\
& =\frac{1}{\alpha}\left[-\Gamma\left(\frac{\alpha+1}{2}\right) \Gamma\left(\frac{2-\alpha}{2}\right) / \Gamma\left(\frac{1}{2}\right) 2^{1-\alpha}+1\right]=-\frac{1}{\alpha}\left[B\left(\frac{\alpha+1}{2}, \frac{2-\alpha}{2}\right) 2^{-\alpha}-1\right] .
\end{aligned}
$$

We thus proved (11) for $\alpha \neq 1$. The case of $\alpha=1$ is trivial. In fact, $\gamma(1,0)=0$.

Lemma 3. Let $-1<r<\alpha<2$ and $\alpha>0$. There exists a constant $c$ such that

$$
\int_{D \backslash B(x, a)} \frac{y_{d}^{r}}{|x-y|^{d+\alpha}} d y \leq c a^{-\alpha}\left(a \vee x_{d}\right)^{r}
$$

for every $a>0$ and $x \in D$.

Proof. Let $B(x, s, t)=B(x, t) \backslash B(x, s)$. If $a \geq x_{d} / 2$ then

$$
\begin{aligned}
\int_{D \backslash B(x, a)} \frac{y_{d}^{r}}{|x-y|^{d+\alpha}} d y & \leq c \sum_{k=0}^{\infty} \int_{D \cap B\left(x, 2^{k} a, 2^{k+1} a\right)} \frac{y_{d}^{r}}{\left(2^{k} a\right)^{d+\alpha}} d y \\
& \leq c^{\prime} \sum_{k=0}^{\infty}\left(2^{k} a\right)^{r-\alpha}=c^{\prime \prime} a^{r-\alpha}
\end{aligned}
$$

If $a<x_{d} / 2$ then

$$
\int_{D \cap B\left(x, a, x_{d}\right)} \frac{y_{d}^{r}}{|x-y|^{d+\alpha}} d y \leq c x_{d}^{r} a^{-\alpha},
$$

and, by first part of the proof,

$$
\int_{D \backslash B\left(x, x_{d}\right)} \frac{y_{d}^{r}}{|x-y|^{d+\alpha}} d y \leq c x_{d}^{r-\alpha} .
$$

This ends the proof.

Recall that $\mathbf{p}=\frac{\alpha-1}{2}$, and $u_{n}$ is defined by (10). 
Lemma 4. There exists a constant $c$ independent of $n$, such that

$$
\int_{D} \int_{D} \frac{\left(u_{n}(x)-u_{n}(y)\right)^{2}}{|x-y|^{d+\alpha}} d y d x \leq c n^{2(d-1)}+2 \kappa_{d, \alpha} \int_{D} u_{n}^{2}(x) x_{d}^{-\alpha} d x .
$$

Proof. To simplify the notation we let $K_{n}=\operatorname{supp} u_{n}$ and $u=u_{n}, v=v_{n}$. By (9) and (6) we have

$$
\begin{aligned}
\int_{D} \int_{D} \frac{(u(x)-u(y))^{2}}{|x-y|^{d+\alpha}} d x d y= & 2 \kappa_{d, \alpha} \int_{D} u^{2}(x) x_{d}^{-\alpha} d x \\
& +\int_{D} \int_{D} \frac{(v(x)-v(y))^{2}}{|x-y|^{d+\alpha}} w(x) w(y) d x d y .
\end{aligned}
$$

We will estimate the latter (double) integral by $c n^{2(d-1)}$, by splitting it into the sum of the following six integrals $I_{1}+\ldots+I_{6}$.

We will first consider the case of $\alpha \geq 1$.

If $x \in K_{n}$ and $y \in B\left(x, \frac{1}{4 n}\right)$, then $|v(x)-v(y)| \leq c|x-y| x_{d}^{-1}$, as follows from (ii) and (iii). We thus have

$$
\begin{aligned}
I_{1} & =\int_{D} \int_{B\left(x, \frac{1}{4 n}\right)} \frac{(v(x)-v(y))^{2}}{|x-y|^{d+\alpha}} w(x) w(y) d y d x \\
& \leq 2 \int_{K_{n}} \int_{B\left(x, \frac{1}{4 n}\right)} \frac{(v(x)-v(y))^{2}}{|x-y|^{d+\alpha}} w(x) w(y) d y d x \\
& \leq c \int_{K_{n}} \int_{B\left(x, \frac{1}{4 n}\right)} \frac{x_{d}^{2 \mathbf{p}-2}}{|x-y|^{d+\alpha-2}} d y d x \\
& \leq c^{\prime} n^{2(d-1)} .
\end{aligned}
$$

A similar argument gives

$$
I_{2}=\int_{\left\{x: x_{d} \geq \frac{1}{2}\right\}} \int_{B\left(x, \frac{1}{4}\right)} \frac{(v(x)-v(y))^{2}}{|x-y|^{d+\alpha}} w(x) w(y) d y d x \leq c n^{2(d-1)} .
$$

We then have by Lemma 3 for $a=1 / 4$ and $r=\mathbf{p}$

$$
\begin{aligned}
I_{3} & =\int_{D} \int_{D \backslash B\left(x, \frac{1}{4}\right)} \frac{(v(x)-v(y))^{2}}{|x-y|^{d+\alpha}} d y d x \\
& \leq \int_{K_{n}} \int_{D \backslash B\left(x, \frac{1}{4}\right)} \frac{c}{|x-y|^{d+\alpha}} w(x) w(y) d y d x \\
& \leq c^{\prime} n^{2(d-1)} .
\end{aligned}
$$

If $d \geq 2$ then we consider $P_{n}=\left\{x \in \mathbb{R}^{d}:\left\|x^{\prime}\right\| \geq n^{2}-1,0<x_{d}<\frac{1}{2}\right\}$ and $P_{n}^{0}=P_{n} \cap\left\{x \in \mathbb{R}^{d}:\left\|x^{\prime}\right\|<n^{2}+\frac{5}{4}\right\}$. We obtain

$$
\begin{aligned}
I_{4} & =\int_{P_{n}} \int_{D \cap B\left(x, \frac{1}{4 n}, \frac{1}{4}\right)} \frac{(v(x)-v(y))^{2}}{|x-y|^{d+\alpha}} w(x) w(y) d y d x \\
& \leq \int_{P_{n}^{0}} \int_{D \backslash B\left(x, \frac{1}{4 n}\right)} \frac{c}{|x-y|^{d+\alpha}} d y d x \\
& \leq c^{\prime}\left|P_{n}^{0}\right| n^{\alpha} \leq c^{\prime \prime} n^{2(d-1)}
\end{aligned}
$$


We let $R_{n}=\left\{x \in \mathbb{R}^{d}:\left\|x^{\prime}\right\|<n^{2}-1,0<x_{d}<\frac{2}{n}\right\}$ if $d \geq 2$, and we let $R_{n}=\left\{x \in \mathbb{R}: 0<x<\frac{2}{n}\right\}$ if $d=1$. We have

$$
\begin{aligned}
I_{5} & =\int_{R_{n}} \int_{D \cap B\left(x, \frac{1}{4 n}, \frac{1}{4}\right)} \frac{(v(x)-v(y))^{2}}{|x-y|^{d+\alpha}} w(x) w(y) d y d x \\
& \leq \int_{R_{n}} \int_{D \backslash B\left(x, \frac{1}{4 n}\right)} \frac{c y_{d}^{\mathbf{p}}\left(\frac{1}{n}\right)^{\mathbf{p}}}{|x-y|^{d+\alpha}} d y d x \leq c^{\prime} n^{2(d-1)} .
\end{aligned}
$$

In the last inequality above we have used Lemma 3 with $a=\frac{1}{4 n}$ and $r=\mathbf{p}$.

We define $L_{n}=\left\{x \in \mathbb{R}: \frac{2}{n} \leq x<\frac{1}{2}\right\}$ in dimension $d=1$, and for $d \geq 2$ we let $L_{n}=\left\{x \in \mathbb{R}^{d}:\left\|x^{\prime}\right\|<n^{2}-1, \frac{2}{n} \leq x_{d}<\frac{1}{2}\right\}$. We have

$$
\begin{aligned}
I_{6} & =\int_{L_{n}} \int_{D \cap B\left(x, \frac{1}{4 n}, \frac{1}{4}\right)} \frac{(v(x)-v(y))^{2}}{|x-y|^{d+\alpha}} w(x) w(y) d y d x \\
& \leq \int_{L_{n}} \int_{\left\{y: 0<y_{d}<\frac{1}{n}\right\}} \frac{w(x) w(y)}{|x-y|^{d+\alpha}} d y d x
\end{aligned}
$$

For $d \geq 2$ and $x \in L_{n}$ we have

$$
\begin{aligned}
& \int_{\left\{y: 0<y_{d}<\frac{1}{n}\right\}} \frac{d y}{|x-y|^{d+\alpha}} \leq c \int_{\left\{y: 0<y_{d}<\frac{1}{n}\right\}} \frac{d y}{\left(\left|x^{\prime}-y^{\prime}\right|^{2}+x_{d}^{2}\right)^{(d+\alpha) / 2}} \\
= & \frac{c}{n}\left(\int_{\left\{y^{\prime} \in \mathbb{R}^{d-1}:\left|x^{\prime}-y^{\prime}\right|<x_{d}\right\}}+\int_{\left\{y^{\prime} \in \mathbb{R}^{d-1}:\left|x^{\prime}-y^{\prime}\right| \geq x_{d}\right\}}\right) \frac{d y^{\prime}}{\left(\left|x^{\prime}-y^{\prime}\right|^{2}+x_{d}^{2}\right)^{(d+\alpha) / 2}} \\
\leq & c^{\prime} \frac{x_{d}^{-\alpha-1}}{n}
\end{aligned}
$$

thus

$$
I_{6} \leq c \int_{L_{n}}\left(\frac{x_{d}}{n}\right)^{\mathbf{p}} \frac{x_{d}^{-\alpha-1}}{n} d x \leq c^{\prime} n^{2(d-1)} .
$$

The case of $d=1$ is left to the reader.

We now consider the case of $\alpha<1$. We have

$$
\begin{aligned}
I= & \int_{D} \int_{D} \frac{(v(x)-v(y))^{2}}{|x-y|^{d+\alpha}} w(x) w(y) d x d y \\
\leq & \int_{D} \int_{B\left(x, \frac{1}{4}\right)}+\int_{\left\{x: x_{d} \geq \frac{n}{2}\right\}} \int_{B\left(x, \frac{n}{4}\right)}+\int_{D} \int_{D \backslash B\left(x, \frac{n}{4}\right)}+\int_{P_{n}} \int_{D \cap B\left(x, \frac{1}{4}, \frac{n}{4}\right)} \\
& +\int_{\left\{x: 0<x_{d}<2\right\}} \int_{D \cap B\left(x, \frac{1}{4}, \frac{n}{4}\right)}+\int_{L_{n}} \int_{D \cap B\left(x, \frac{1}{4}, \frac{n}{4}\right)} \\
= & I_{1}+I_{2}+I_{3}+I_{4}+I_{5}+I_{6},
\end{aligned}
$$

where

$$
\begin{aligned}
& P_{n}=\left\{x \in \mathbb{R}^{d}:\left\|x^{\prime}\right\| \geq n^{2}-n, 0<x_{d}<\frac{n}{2}\right\} ; \\
& L_{n}=\left\{x \in \mathbb{R}^{d}:\left\|x^{\prime}\right\|<n^{2}-n, 2 \leq x_{d}<\frac{n}{2}\right\},
\end{aligned}
$$

for $d \geq 2$, and $P_{n}=\emptyset, L_{n}=\left(2, \frac{n}{2}\right)$ for $d=1$. We estimate the integrals $I_{k}$ in a similar way as for $\alpha \geq 1$. The details are left to the reader. 
Similar but simpler estimates were given in [16 to prove that the Hardy constant of a bounded Lipschitz domain (e.g. of an interval) is zero if $\alpha \leq 1$. We also like to mention that there is an alternative proof of Lemma 4 (not given here), which explicitly uses the fact that that $w^{2}(x)=x_{d}^{\alpha-1}$ is harmonic ([7]) for $\Delta_{D}^{\alpha / 2}$. Similarly, the best constant, $1 / 4$, in the classical Hardy inequality for the half-space $D$ is obtained by considering $w(x)=\sqrt{x_{d}}$ in Fitzsimmons' ratio $\nu=-\Delta w / w$.

Acknowledgement. We thank Grzegorz Karch for a discussion on recent results on non-local operators, and Rodrigo Bañuelos, Małgorzata Kuchta and Jacek Zienkiewicz for discussions on classical Hardy inequality.

\section{REFERENCES}

[1] A. Ancona. On strong barriers and an inequality of Hardy for domains in $\mathbf{R}^{n}$. J. London Math. Soc. (2), 34(2):274-290, 1986.

[2] R. Bañuelos, M. van den Berg, and T. Carroll. Torsional rigidity and expected lifetime of Brownian motion. J. London Math. Soc. (2), 66(2):499-512, 2002.

[3] G. Barles, E. Chasseigne, and C. Imbert. On the dirichlet problem for second-order elliptic integro-differential equations. Indiana Univ. Math. J., 2008.

[4] W. Beckner. Pitt's inequality with sharp convolution estimates. Proc. Amer. Math. Soc., 136(5):1871-1885, 2008.

[5] P. R. Beesack. Hardy's inequality and its extensions. Pacific J. Math., 11:39-61, 1961.

[6] K. Bogdan. The boundary Harnack principle for the fractional Laplacian. Studia Math., 123(1):43-80, 1997.

[7] K. Bogdan, K. Burdzy, and Z.-Q. Chen. Censored stable processes. Probab. Theory Related Fields, 127(1):89-152, 2003.

[8] K. Bogdan, T. Kulczycki, and M. Kwaśnicki. Estimates and structure of $\alpha$-harmonic functions. Probab. Theory Related Fields, 140(3-4):345-381, 2008.

[9] K. Bogdan and P. Sztonyk. Harnack's inequality for stable Lévy processes. Potential Anal., 22(2):133-150, 2005.

[10] K. Bogdan and P. Sztonyk. Estimates of the potential kernel and Harnack's inequality for the anisotropic fractional Laplacian. Studia Math., 181(2):101-123, 2007.

[11] H. Brezis and M. Marcus. Hardy's inequalities revisited. Ann. Scuola Norm. Sup. Pisa Cl. Sci. (4), 25(1-2):217-237 (1998), 1997. Dedicated to Ennio De Giorgi.

[12] K. Brezis. How to recognize constant functions. A connection with Sobolev spaces. Uspekhi Mat. Nauk, 57(4(346)):59-74, 2002.

[13] Z.-Q. Chen and R. Song. Martin boundary and integral representation for harmonic functions of symmetric stable processes. J. Funct. Anal., 159(1):267-294, 1998.

[14] Z.-Q. Chen and R. Song. Hardy inequality for censored stable processes. Tohoku Math. J. (2), 55(3):439-450, 2003.

[15] E. B. Davies. A review of Hardy inequalities. In The Maz'ya anniversary collection, Vol. 2 (Rostock, 1998), volume 110 of Oper. Theory Adv. Appl., pages 55-67. Birkhäuser, Basel, 1999.

[16] B. Dyda. A fractional order Hardy inequality. Ill. J. Math., 48(2):575-588, 2004.

[17] B. Dyda. On comparability of integral forms. J. Math. Anal. Appl., 318(2):564-577, 2006.

[18] J. Dziubański and J. Zienkiewicz. Hardy spaces $H^{1}$ for Schrödinger operators with certain potentials. Studia Math., 164(1):39-53, 2004.

[19] P. J. Fitzsimmons. Hardy's inequality for Dirichlet forms. J. Math. Anal. Appl., 250(2):548560, 2000.

[20] B. Florkiewicz and M. Kuchta. Some quadratic integral inequalities of first order. Colloq. Math., 75(1):7-18, 1998.

[21] R. Frank, E. Lieb, and R. Seiringer. Hardy-lieb-thirring inequalities for fractional schrödinger operators. http://arxiv.org/list/math.SP/0610593, 2006.

[22] M. Fukushima, Y. Ōshima, and M. Takeda. Dirichlet forms and symmetric Markov processes, volume 19 of de Gruyter Studies in Mathematics. Walter de Gruyter \& Co., Berlin, 1994.

[23] N. Ghoussoub and A. Moradifam. On the best possible remaining term in the Hardy inequality, 2007. 
[24] Q. Guan. Boundary Harnack inequalities for regional fractional Laplacian, 2007.

[25] Q.-Y. Guan. Integration by parts formula for regional fractional Laplacian. Comm. Math. Phys., 266(2):289-329, 2006.

[26] Q.-Y. Guan and Z.-M. Ma. Reflected symmetric $\alpha$-stable processes and regional fractional Laplacian. Probab. Theory Related Fields, 134(4):649-694, 2006.

[27] H. P. Heinig, A. Kufner, and L.-E. Persson. On some fractional order Hardy inequalities. J. Inequal. Appl., 1(1):25-46, 1997.

[28] I. W. Herbst. Spectral theory of the operator $\left(p^{2}+m^{2}\right)^{1 / 2}-Z e^{2} / r$. Comm. Math. Phys., 53(3):285-294, 1977.

[29] N. Jacob. Pseudo differential operators and Markov processes. Vol. I. Imperial College Press, London, 2001. Fourier analysis and semigroups.

[30] N. Jacob and R. L. Schilling. Lévy-type processes and pseudodifferential operators. In Lévy processes, pages 139-168. Birkhäuser Boston, Boston, MA, 2001.

[31] T. Jakubowski. The estimates for the Green function in Lipschitz domains for the symmetric stable processes. Probab. Math. Statist., 22(2, Acta Univ. Wratislav. No. 2470):419-441, 2002.

[32] P. Kim. Weak convergence of censored and reflected stable processes. Stochastic Process. Appl., 116(12):1792-1814, 2006.

[33] N. Krugljak, L. Maligranda, and L. E. Persson. On an elementary approach to the fractional Hardy inequality. Proc. Amer. Math. Soc., 128(3):727-734, 2000.

[34] A. Kufner and L.-E. Persson. Integral inequalities with weights. Academia, Prague, 2000.

[35] Z. M. Ma and M. Röckner. Introduction to the theory of (nonsymmetric) Dirichlet forms. Universitext. Springer-Verlag, Berlin, 1992.

[36] M. Marcus, V. J. Mizel, and Y. Pinchover. On the best constant for Hardy's inequality in $\mathbf{R}^{n}$. Trans. Amer. Math. Soc., 350(8):3237-3255, 1998

[37] K. Michalik and M. Ryznar. Relative Fatou theorem for $\alpha$-harmonic functions in Lipschitz domains. Illinois J. Math., 48(3):977-998, 2004.

[38] R. L. Schilling and T. Uemura. On the Feller property of Dirichlet forms generated by pseudo differential operators. Tohoku Math. J. (2), 59(3):401-422, 2007.

[39] D. Yafaev. Sharp constants in the Hardy-Rellich inequalities. J. Funct. Anal., 168(1):121-144, 1999.

Institute of Mathematics and Computer Science, Wroceaw University of Technology, Wybrzeże Wyspiańskiego 27, 50-370 Wroceaw, Poland

E-mail address: bogdan@pwr.wroc.pl bdyda@pwr.wroc.pl 